\title{
AMP-activated protein kinase inhibits IL-6-stimulated inflammatory response in human liver cells by suppressing phosphorylation of signal transducer and activator of transcription 3 (STAT3)
}

\author{
A. Nerstedt • A. Johansson • C. X. Andersson • \\ E. Cansby • U. Smith • M. Mahlapuu
}

Received: 21 April 2010 / Accepted: 24 June 2010 / Published online: 24 July 2010

(C) Springer-Verlag 2010

\begin{abstract}
Aim/hypothesis The aim of the study was to examine the possible role of AMP-activated protein kinase (AMPK) in the regulation of the inflammatory response induced by cytokine action in human liver cells.

Methods IL-6-stimulated expression of the genes for acutephase response markers serum amyloid A (SAA1, SAA2) and haptoglobin $(H P)$ in the human hepatocarcinoma cell line HepG2 were quantified after modulation of AMPK activity by pharmacological agonists (5-amino-4-imidazolecarboxamideriboside [AICAR], metformin) or by using small interfering (si) RNA transfection. The intracellular signalling pathway mediating the effect of AMPK on IL-6stimulated acute-phase marker expression was characterised by assessing the phosphorylation levels of the candidate protein signal transducer and activator of transcription 3 (STAT3) in response to AMPK agonists.

Results AICAR and metformin markedly blunt the IL-6stimulated expression of $S A A$ cluster genes as well as of haptoglobin in a dose-dependent manner. Moreover, the repression of AMPK activity by siRNA significantly
\end{abstract}

Electronic supplementary material The online version of this article (doi:10.1007/s00125-010-1856-z) contains supplementary material, which is available to authorised users.

A. Nerstedt · A. Johansson · C. X. Andersson · E. Cansby ·

U. Smith $\cdot$ M. Mahlapuu $(\bowtie)$

The Lundberg Laboratory for Diabetes Research,

Center of Excellence for Cardiovascular and Metabolic Research,

Department of Molecular and Clinical Medicine/Diabetes,

The Sahlgrenska Academy, University of Gothenburg,

Blå stråket 5 ,

SE-413 45 Göteborg, Sweden

e-mail: margit.mahlapuu@gu.se reversed the inhibition of $S A A$ expression by both AICAR and metformin, indicating that the effect of the agonists is dependent on AMPK. For the first time we show that AMPK appears to regulate IL-6 signalling by directly inhibiting the activation of the main downstream target of IL-6, STAT3.

Conclusions/interpretation We provide evidence for a key function of AMPK in suppression of the acute-phase response caused by the action of IL- 6 in liver, suggesting that AMPK may act as an intracellular link between chronic low-grade inflammation and metabolic regulation in peripheral metabolic tissues.

Keywords AMP-activated protein kinase - Inflammation * Interleukin-6 6 Liver Type 2 diabetes

$\begin{array}{ll}\text { Abbreviations } \\ \text { ACC } & \text { Acetyl-CoA carboxylase } \\ \text { AICAR } & \text { 5-Amino-4-imidazole-carboxamideriboside } \\ \text { AMPK } & \text { AMP-activated protein kinase } \\ \text { CRP } & \text { C-reactive protein } \\ \text { HRP } & \text { Horseradish peroxidase } \\ \text { LPS } & \text { Lipopolysaccharide } \\ \text { MTT } & \text { 3-(4,5-Dimethylthiazol-2-yl)-2,5- } \\ & \text { diphenyltetrazolium bromide } \\ \text { qRT-PCR } & \text { Quantitative RT-PCR } \\ \text { SAA } & \text { Serum amyloid A } \\ \text { siRNA } & \text { Small interfering RNA } \\ \text { SOCS3 } & \text { Suppressor of cytokine signalling 3 } \\ \text { STAT3 } & \text { Signal transducer and activator of } \\ & \text { transcription 3 }\end{array}$




\section{Introduction}

Type 2 diabetes is characterised by hyperinsulinaemia, hyperglycaemia and dyslipidaemia. Weight gain and obesity are the major risk factors for development of insulin resistance, a key feature in the aetiology of type 2 diabetes. Recent evidence indicates that excessive caloric intake and obesity lead to chronic low-grade inflammation in metabolic tissues, which further promotes insulin resistance (reviewed by Hotamisligil [1]). In the state of metabolic disease, the increased secretion of proinflammatory cytokines such as IL-6, TNF- $\alpha$ and IL$1 \beta$ from adipocytes and macrophages initiates the activation of inflammatory pathways in insulin target cells, ultimately leading to decreased insulin sensitivity (reviewed by Wellen and Hotamisligil [2]). However, the intracellular mediators of the negative regulation of insulin signalling by inflammatory cytokines are only partly understood.

AMP-activated protein kinase (AMPK) has long been identified as a critical regulator of energy homeostasis in metabolic tissues, but evidence suggesting its possible role in the regulation of inflammatory signalling is only starting to emerge. AMPK is a heterotrimeric serine/ threonine protein kinase composed of a catalytic $\alpha$ subunit and non-catalytic $\beta$ and $\gamma$ subunits, all of which are required for its activity. The mammalian genome contains seven AMPK genes encoding for two $\alpha$, two $\beta$ and three $\gamma$ isoforms. The catalytic $\alpha$ subunit contains a conventional serine/threonine protein kinase domain, and phosphorylation of the $\mathrm{Thr}^{172}$ residue within the activation loop of the $\alpha$ subunit by upstream kinases is essential for the activity of the heterotrimer (reviewed by Carling [3]). Two kinases have been established as upstream activators of AMPK: the protein kinase serine/threonine 11 (LKB1)/ STE20-related kinase adaptor alpha (STRAD)/calcium binding protein 39 (MO25) complex [4] and the calcium/ calmodulin-dependent protein kinase kinase $\beta$ (CAMKK $\beta)$ [5]. Once phosphorylated at $\mathrm{Thr}^{172}$, AMPK can further be activated by allosteric binding of AMP to the regulatory $\gamma$ subunit [6]. Activation of AMPK increases glucose uptake and lipid oxidation in the skeletal muscle, inhibits glucose and lipid synthesis and enhances lipid oxidation in the liver, and reduces lipolysis and lipogenesis in adipose tissue, all together resulting in a favourable metabolic milieu, i.e. decreased plasma glucose and lipid levels [7].

A possible role for AMPK in regulation of the inflammatory response has been suggested based on studies using pharmacological agonists. The AMPK activator, 5-amino-4-imidazole-carboxamideriboside (AICAR), has been demonstrated to reduce the severity of airway inflammation in murine experimental models of asthma and lipopolysaccharide (LPS)-induced acute lung injury $[8,9]$. Moreover, the AMPK agonist metformin, currently the most widely prescribed oral blood glucose- lowering agent, has been reported to decrease the levels of plasma inflammatory indices, such as C-reactive protein (CRP) [10]. Another well-characterised AMPK agonist, rosiglitazone, has been shown to cause a fall in plasma concentrations of inflammatory signals such as CRP and serum amyloid A (SAA), in addition to its established role in regulating glucose homeostasis [11]. Interestingly, both metformin and AICAR attenuated the TNF- $\alpha$-induced expression of proinflammatory and cell adhesion molecules in human endothelial cells via activation of AMPK [12]. AICAR also decreased inflammatory mediator production in stimulated kidney mesangial cells in an AMPK-dependent manner [13]. The anti-inflammatory effect of AICAR in macrophages was shown to be independent of AMPK [14, 15]. However, by using transfection of macrophages with a constitutively active and dominant negative form of AMPK $\alpha 1$, it was recently shown that activation of AMPK suppresses proinflammatory responses and promotes macrophage polarisation towards an anti-inflammatory functional phenotype [16]. In different cell types, stimulation by inflammatory mediators such as macrophage migration inhibitory factor, ciliary neurotrophic factor and IL-6 correlates with AMPK activation [17-20], further supporting a possible cross-talk between these pathways.

We investigated the function of AMPK in regulating IL6-stimulated inflammatory signalling in liver cells. We provide evidence for a clear role of AMPK in suppressing the proinflammatory responses caused by the action of IL-6 in liver, suggesting that AMPK may act as an intracellular link between inflammation and metabolic regulation in peripheral metabolic tissues. Moreover, for the first time we report that AMPK regulates IL-6 signalling by directly inhibiting the activation of the main downstream target of IL-6, signal transducer and activator of transcription 3 (STAT3).

\section{Methods}

Reagents Human recombinant (r) IL-1 $\beta$, rIL-6, rTNF- $\alpha$ and mouse rIL-6 were purchased from Sigma Aldrich (St Louis, MO, USA). Western blot detection of specific proteins used the following primary antibodies: antiphospho-AMPK $\alpha\left(\mathrm{Thr}^{172}\right.$, \#2531; Cell Signaling Technology, Boston, MA, USA), anti-AMPK $\alpha$ (\#2532; Cell Signaling Technology), anti-phospho-acetyl-CoA carboxylase (ACC; $\mathrm{Ser}^{79}$, \#3661; Cell Signaling Technology), anti-ACC (\#3662; Cell Signaling Technology), antiphospho-STAT3 (Tyr $^{705}$, \#9138; Cell Signaling Technology), anti-STAT3 (610189; Becton Dickinson, Franklin Lakes, NJ, USA), anti-LKB1 (\#3047S, Cell Signaling Technology) and horseradish peroxidase (HRP)-conjugated 
secondary antibodies anti-rabbit IgG (\#7074; Cell Signaling Technology), and anti-mouse IgG (\#7076; Cell Signaling Technology). Antibodies against AMPK $\alpha 1$ and AMPK $\alpha 2$ subunits were custom designed and have been described previously [21]. AICAR and metformin (1,1-dimethyl biguanide hydrochloride) were purchased from Sigma Aldrich.

Cytokine stimulation of HepG2 cells The HepG2 cells (hepatocellular carcinoma, human, American Type Culture Collection, Manassas, VA, USA) were maintained in DMEM (Lonza, Basel, Switzerland) including $4.5 \mathrm{~g} / 1$ glucose and L-glutamine, supplemented with $10 \%$ (vol./vol.) FBS (Invitrogen, San Diego, CA, USA), penicillinstreptomycin $(1 \times$; Invitrogen) and non-essential amino acids (1×, PAA Laboratories, Pasching, Austria). The cell density was adjusted to $2 \times 10^{5}$ cells $/ \mathrm{ml}$ and $1 \mathrm{ml}$ of the cell suspension was added per well to 12 -well cell culture plates (NUNC, Roskilde, Denmark). Either when the treatment with AICAR or metformin was initiated or $16 \mathrm{~h}$ before cytokine stimulation, the medium was exchanged for DMEM containing $1 \mathrm{~g} / 1$ glucose, supplemented with $0.5 \%$ (wt/vol.) cell-culture-tested BSA (Sigma Aldrich). Cells were treated with AICAR or metformin at the concentrations and times indicated, followed by stimulation with human $\mathrm{rIL}-1 \beta(10 \mathrm{ng} / \mathrm{ml})$, rIL-6 (10 $\mathrm{ng} / \mathrm{ml})$ or $\mathrm{rTNF}-\alpha$ $(10 \mathrm{ng} / \mathrm{ml})$ for $6 \mathrm{~h}$.

Cytokine stimulation in isolated primary hepatocytes Hepatocytes were isolated from mice using a two-step collagenase perfusion method [22] and seeded on collagencoated six-well plates (Falcon Primaria, Becton Dickinson) at a density of 1 million cells/well. The cells were cultured in Williams E medium (Invitrogen) supplemented with $0.28 \mathrm{~mol} / 1$ sodium ascorbate (Sigma Aldrich), $0.1 \mathrm{mmol} / 1$ sodium selenite (Sigma Aldrich), pencillin-streptomycin (1×; Invitrogen), $3 \mathrm{~g} / 1$ glucose (Sigma Aldrich) and $26 \mathrm{IU} / 1$ insulin (Actrapid Penfill; Novo Nordisk, Bagsværd, Denmark). Cells were treated with AICAR $(2 \mathrm{mmol} / \mathrm{l})$ or metformin $(5 \mathrm{mmol} / \mathrm{l})$ for $16 \mathrm{~h}$ followed by stimulation with mouse rIL-6 (20 ng/ml, Sigma Aldrich) for $20 \mathrm{~min}$.

Western blot analysis Cells were washed in PBS and lysis buffer $(25 \mathrm{mmol} / 1 \mathrm{Tris}-\mathrm{HCl}$, pH 7.4, $0.5 \mathrm{mmol} / \mathrm{l}$ EGTA, $25 \mathrm{mmol} / \mathrm{l} \mathrm{NaCl}, 1 \%$ (vol./vol.) Nonidet P-40, $10 \mathrm{mmol} / 1$ $\mathrm{NaF}, 1 \mathrm{mmol} / \mathrm{l}$ orthovanadate, $100 \mathrm{nmol} / \mathrm{l}$ okadaic acid, and Complete Protease Inhibitor Cocktail (Roche Applied Science, Mannheim, Germany) was then added to the cells, and the lysate was centrifuged at $20,000 \mathrm{~g}$ for $10 \mathrm{~min}$. Protein concentration was measured using the BCA protein assay kit (Thermo Scientific, Rockford, IL, USA). The lysate was boiled in SDS loading buffer and applied on $7.5 \%, 10 \%$ or $12 \%$ SDS-PAGE (Lonza, Rockland, ME,
USA). Membranes were blocked in $10 \mathrm{mmol} / 1$ Tris base, $150 \mathrm{mmol} / \mathrm{l} \mathrm{NaCl}$ and $0.25 \%$ (vol./vol.) Tween 20 (TBST buffer) containing $5 \%$ (wt/vol.) low-fat milk powder for $1 \mathrm{~h}$ at room temperature. Membranes were then incubated with primary antibodies overnight at $4{ }^{\circ} \mathrm{C}$ and washed with TBST buffer followed by incubation with the appropriate HRP-conjugated secondary antibody for $1 \mathrm{~h}$ at room temperature. Immunoreactive proteins were visualised by enhanced chemiluminescence (ECL Western Blotting detection reagent, GE Healthcare, Amersham, UK; or ImmunSTAR HRP Chemiluminescent Kit, Bio-Rad, Richmond, CA, USA).

Quantitative real-time PCR RNA was isolated with the RNeasy Mini Kit (Qiagen, Valencia, CA, USA), and the following reverse transcriptase PCR for cDNA synthesis was made using the High Capacity cDNA Reverse Transcription Kit (Applied Biosystems, Foster City, CA, USA). Relative quantification was performed using the ABI Prism 7900HT Sequencing Detection System (Applied Biosystems). Primers and probes were designed using PRIMER EXPRESS software (Applied Biosystems), and sequences are available upon request. Relative quantities of target transcripts were calculated from duplicate samples after normalisation of the data against the endogenous control, human 18S rRNA (Applied Biosystems).

Cell viability assessment The cell viability was estimated using TACS 3-(4,5-dimethylthiazol-2-yl)-2,5-diphenyltetrazolium bromide (MTT) assay following the manufacturer's instructions (R\&D Systems, Minneapolis, MN, USA). Briefly, $16 \mathrm{~h}$ after addition of the indicated concentrations of AICAR or metformin, MTT reagent was added to the HepG2 cells in 96-well plates and incubated for $4 \mathrm{~h}$ at $37^{\circ} \mathrm{C}$. Then detergent reagent was added and incubated for $6 \mathrm{~h}$ at $37^{\circ} \mathrm{C}$ before the absorbance was measured using the Versamax microplate reader (Molecular Devices, Sunnyvale, CA, USA) at $570 \mathrm{~nm}$ with a reference wavelength of $650 \mathrm{~nm}$.

RNA interference HepG2 cells were transfected with siRNA directed against AMPK $\alpha 1$ (PRKKA1, \#AM51334; Ambion, Austin, TX, USA) and AMPK $\alpha 2$ (sc-38923; Santa Cruz Biotechnology, Santa Cruz, CA, USA) subunits, LKB1 (s13581, Ambion) or scrambled siRNA (\#AM4635, Ambion) as a control. Briefly, cells were transfected with $30 \mathrm{nmol} / 1$ of total siRNA by using Lipofectamine RNAiMax according to the manufacturer's instructions for reverse transfection (Invitrogen).

Statistical analysis Statistical significance between the groups was calculated with an unpaired Student's $t$ test, with a value of $p<0.05$ considered statistically significant. 
a

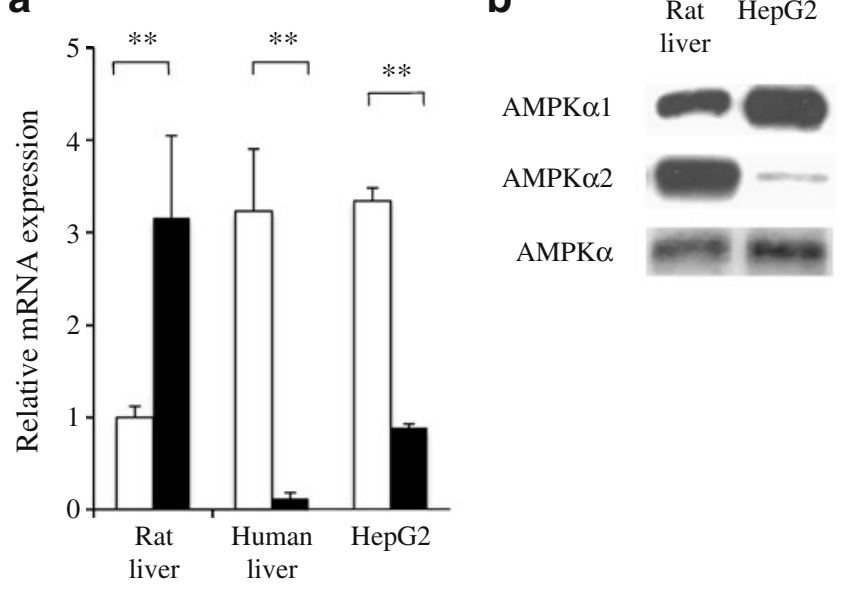

Fig. 1 HepG2 cells produce both AMPK catalytic subunits. a qRTPCR analysis of $A M P K \alpha 1$ (white bars) and $A M P K \alpha 2$ (black bars) mRNA expression in human and rat liver, and HepG2 cells. Data shown as means \pm SEM of three to five independent samples analysed $(* * p<0.01)$. The expression level of $A M P K \alpha 1$ in rat liver is set to 1 . b Lysates of rat liver, as well as HepG2 cells were analysed by western blot using anti-AMPK $\alpha 1$, anti-AMPK $\alpha 2$ and total anti-AMPK $\alpha$ antibodies. Results are representative of three independent analyses with similar results

\section{Results}

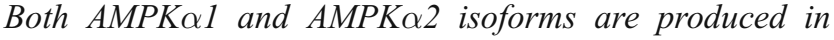
HepG2 cells Previously it has been demonstrated that in the rodent liver, the AMPK $\alpha 1$ - and AMPK $\alpha 2$-containing complexes account for about half of total AMPK activity each [23]. In human liver, and in the human hepatocyte cell line HepG2, the abundance of the two isoforms of the catalytic subunit has not been previously described. Analysis of $A M P K \alpha 1$ (also known as PRKAA1)/AMPK 22 (also known as $P R K A A 2$ ) mRNA expression by quantitative (q) RT-PCR revealed that the mRNA expression level of $A M P K \alpha 1$ was significantly higher compared with $A M P K \alpha 2$ both in adult human liver as well as in HepG2 cells, which was the opposite of the pattern seen in adult rat liver (Fig. 1a). Western blot analysis confirmed that the signal for AMPK $\alpha 2$ was markedly stronger in rodent liver compared with HepG2 cells, whereas the opposite ratio was seen for AMPK $\alpha 1$ (Fig. 1b). As the protein for both catalytic subunits of AMPK was produced in human liver cells, siRNAs against both AMPK $\alpha 1$ and AMPK $\alpha 2$ were combined in our subsequent experiments to impair AMPK activity in HepG2 cells.

AMPK agonists AICAR and metformin repress cytokineinduced expression of inflammatory markers in human liver cells A prominent feature of inflammatory reactions is the induction of acute-phase protein synthesis in hepatocytes. Cytokines, such as IL- $1 \beta$, TNF- $\alpha$, but mainly IL-6, stimulate the production of acute-phase proteins such as SAA and haptoglobin $[24,25]$. To study the ability of a

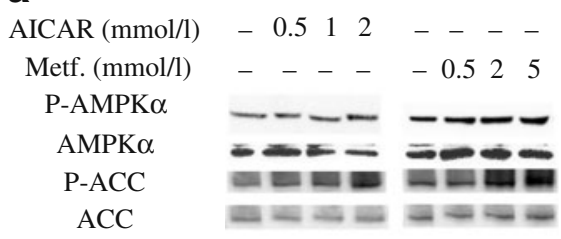

b

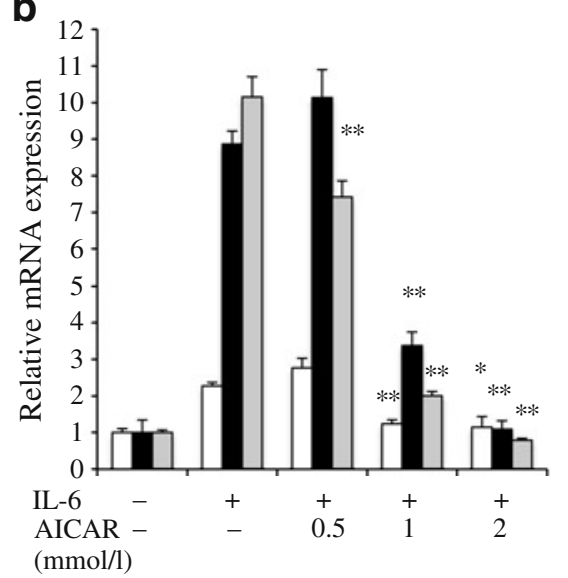

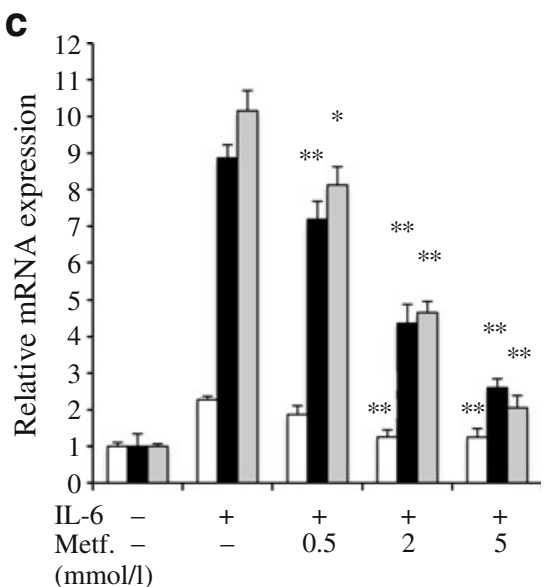

Fig. 2 AICAR and metformin inhibit IL-6-stimulated synthesis of inflammation markers in HepG2 cells in a dose-dependent manner. a Effect of AICAR and metformin on AMPK $\alpha$ and ACC phosphorylation (P-) in HepG2 cells. Cells were cultured with the indicated concentrations of AICAR or metformin for $16 \mathrm{~h}$. Cell lysates were analysed by western blot using antibodies specific for phosphoAMPK $\alpha\left(\mathrm{Thr}^{172}\right)$, total AMPK $\alpha$, phospho-ACC $\left(\mathrm{Ser}^{79}\right)$ or total ACC. Representative western blot of two independent experiments is shown. b, c HepG2 cells were treated with the indicated concentrations of AICAR (b) or metformin (c) for $16 \mathrm{~h}$ followed by IL-6 stimulation for $6 \mathrm{~h}$. Levels of the inflammatory markers $S A A 1$ (white bars), $S A A 2$ (black bars) and haptoglobin (grey bars) were determined by qRT-
PCR. Results are shown as means \pm SEM of three to six determinations with the basal expression of the corresponding marker set to 1 . The data shown are representative of two independent experiments with similar results. ${ }^{*} p<0.05,{ }^{* *} p<0.01$ comparing IL-6 and AICAR or IL-6 and metformin treatment with IL-6-treated cells only. IL-6 significantly increased expression of all acute-phase markers compared with the basal state $(p<0.05$ for $S A A 1, p<0.01$ for $S A A 2$ and haptoglobin). In our hands, considerable inter-assay variability was observed in the fold-induction of acute-phase markers by IL- 6 and correspondingly, the direct comparisons of fold change values within rather than between experiments should be performed. Metf, metformin 
AMPK activation to impair the cytokine-induced inflammatory response in liver, the HepG2 cells were pretreated with different concentrations of AMPK agonists, AICAR or metformin, and then exposed to IL-6 $(10 \mathrm{ng} / \mathrm{ml})$. AICAR is a cell-permeable compound that has been shown to activate AMPK in various tissues, including human liver cells. Once taken up by the cell, AICAR is phosphorylated to 5aminoimidazole-4-carboxamide riboside monophosphate and mimics the allosteric effect of AMP on AMPK [26].
Metformin inhibits enzymatic activity of complex I of the respiratory chain and hence lowers the cellular ATP:ADP ratio, which leads to the activation of AMPK [27]. We assessed the ability of AICAR and metformin to activate AMPK in HepG2 cells by measuring phosphorylation of $\mathrm{Thr}^{172}$ in the AMPK catalytic $\alpha$ subunit, after incubation with increasing concentrations of the agonists. In addition, we measured the levels of $\mathrm{Ser}^{79}$ phosphorylation of ACC, a downstream target of AMPK. Treatment with AICAR and a

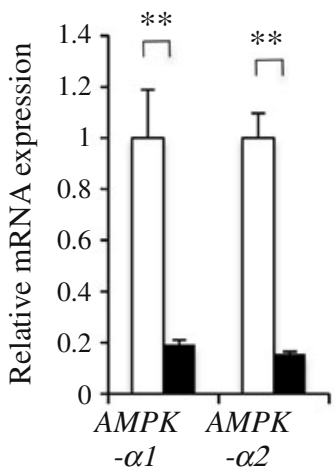

C

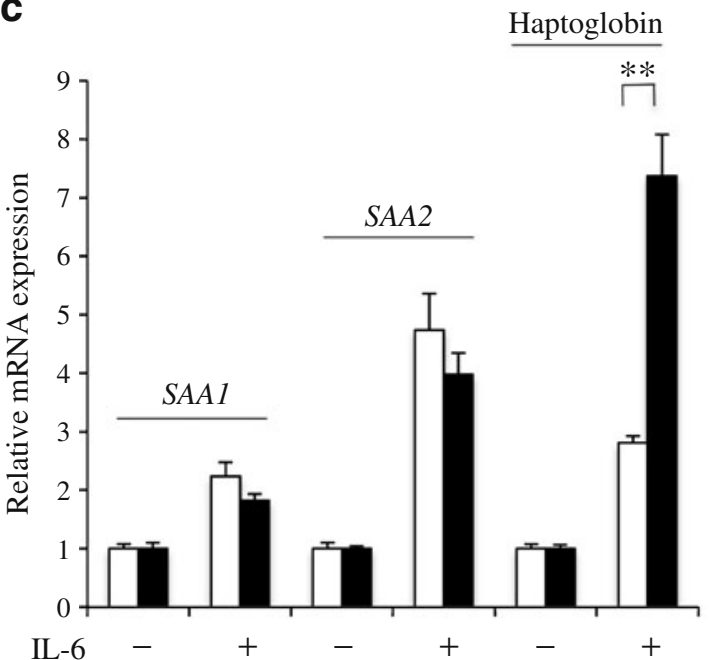

Fig. 3 AICAR and metformin inhibit IL-6-stimulated synthesis of inflammation markers in HepG2 cells in an AMPK-dependent manner. a, b Cells were transfected with $A M P K \alpha 1 / \alpha 2$ siRNA or control siRNA. $72 \mathrm{~h}$ post-transfection, the cells were lysed for qRT-PCR (a) or western blot analysis (b). In (b) AICAR $(1 \mathrm{mmol} / \mathrm{l})$ or metformin $(2 \mathrm{mmol} / \mathrm{l})$ treatment was performed for $16 \mathrm{~h}$ before the harvest. a qRT-PCR analysis of $A M P K \alpha 1$ and $A M P K \alpha 2$ mRNA expression in cells transfected with $A M P K \alpha 1 / \alpha 2$ siRNA (black bars) or control siRNA (white bars). Data shown as means \pm SEM of triplicate determinations $(* * p<0.01)$ from one of five independent experiments with similar results. The expression level of $A M P K \alpha 1$ and $A M P K \alpha 2$ in cells transfected with control siRNA is set to 1 . b Representative western blot analysis of two to four independent experiments using antibodies specific for phospho-AMPK $\alpha\left(\mathrm{Thr}^{172}\right)$, AMPK $\alpha 1$, $\mathrm{AMPK} \alpha 2$, total AMPK $\alpha$, phospho-ACC $\left(\mathrm{Ser}^{79}\right)$, total ACC or b

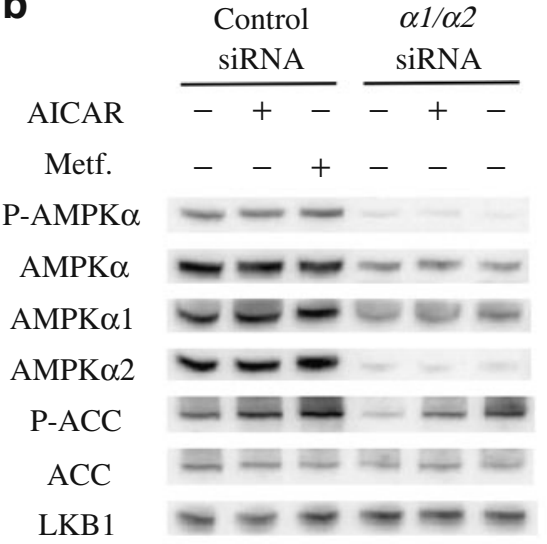

d

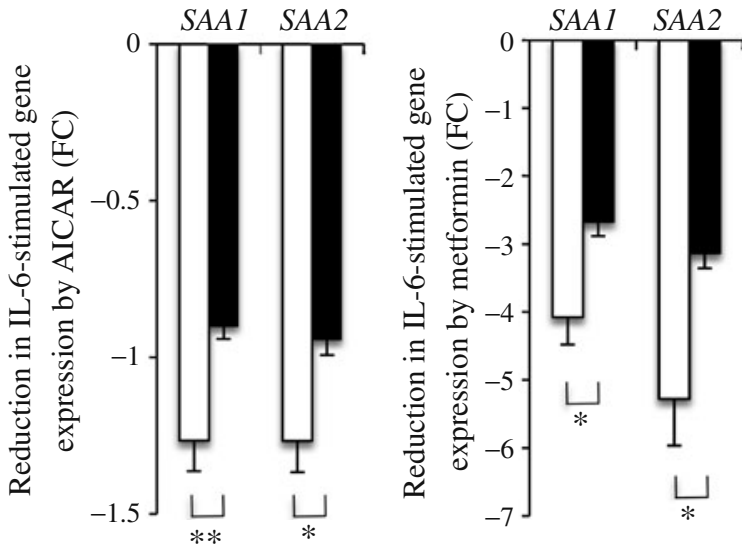

LKB1. c Relative expression level of $S A A 1, S A A 2$ and haptoglobin in response to IL-6 induction $(6 \mathrm{~h})$ determined by qRT-PCR analysis in cells transfected with $A M P K \alpha 1 / \alpha 2$ siRNA (black bars) or control siRNA (white bars). Data shown as means \pm SEM of four determinations with the basal expression of the corresponding maker set to 1 $\left({ }^{* *} p<0.01\right)$. d, e HepG2 cells transfected with $A M P K \alpha 1 / \alpha 2$ siRNA (black bars) or control siRNA (white bars) were treated with AICAR $(1 \mathrm{mmol} / \mathrm{l}$, d) or metformin $(2 \mathrm{mmol} / \mathrm{l}$, e) for $16 \mathrm{~h}$ followed by IL-6 stimulation for $6 \mathrm{~h}$. Reduction in IL-6-stimulated expression of $S A A 1$ and $S A A 2$ in response to AICAR or metformin in cells transfected with $A M P K \alpha 1 / \alpha 2$ siRNA or control siRNA is shown as the fold change of expression (means \pm SEM of three to six determinations) relative to the placebo-treated controls. Similar results were obtained in two independent experiments ${ }^{*} p<0.05$; $* * p<0.01)$. FC, fold change 
metformin dose-dependently increased AMPK $\alpha$ and ACC phosphorylation, an effect that reached a maximum with the highest concentrations of 2 and $5 \mathrm{mmol} / \mathrm{l}$, respectively (Fig. 2a).

As previously described, IL-6 significantly increased the expression of mRNA for $S A A 1, S A A 2$ and haptoglobin in HepG2 cells [24, 25] (Fig. 2b, c). In this study, $16 \mathrm{~h}$ preincubation with AICAR and metformin markedly inhibited the IL-6-induced expression of $S A A 1, S A A 2$ and haptoglobin in a dose-dependent manner (Fig. 2b, c). Moreover, the close-to-maximal activation of AMPK by AICAR and metformin was observed already after 2 or $4 \mathrm{~h}$ of incubation, respectively, and the short-term pretreatment of cells with these agonists repressed the IL-6-stimulated expression of $S A A 1, S A A 2$ and haptoglobin in a similar manner to the treatment for $16 \mathrm{~h}$ (see Electronic supplementary material [ESM] Fig. 1).

As in repression of IL-6-induced gene expression, AICAR ( $2 \mathrm{mmol} / \mathrm{l})$ and metformin $(5 \mathrm{mmol} / \mathrm{l})$ inhibited the expression of $S A A 1$ and $S A A 2$ induced by IL- $1 \beta(10 \mathrm{ng} / \mathrm{ml})$ or TNF- $\alpha$ $(10 \mathrm{ng} / \mathrm{ml})$. The IL-1 $\beta$ or TNF- $\alpha$ failed to induce the expression of haptoglobin in HepG2 cells (data not shown). The indicated concentrations of metformin had no effect on the viability of the HepG2 cells as tested by the MTT assay. All three concentrations of AICAR reduced cell viability by approximately $20-30 \%$ without any dose-response relationship (data not shown). Therefore, it was concluded that the reduction in gene expression observed in response to AICAR or metformin was not related to the reduced cell viability.

AICAR and metformin inhibit inflammatory gene expression in liver by activation of AMPK To further elucidate the role of AMPK in regulating cytokine-stimulated inflammatory responses in liver cells, the AMPK expression was repressed with siRNAs against the catalytic AMPK $\alpha 1$ and AMPK $\alpha 2$ subunits, both expressed in HepG2 cells (Fig. 1). Typically, transfection with siRNA decreased the mRNA expression of both $A M P K \alpha 1$ and $A M P K \alpha 2$ by approximately $80 \%$, compared with control siRNA-transfected cells (Fig. 3a), which was confirmed at the protein level (Fig. 3b). Next, we examined the effect of the $A M P K \alpha 1 / \alpha 2$ siRNA on cytokine-mediated inflammatory gene expression. The siRNA against AMPK $\alpha$ subunits had no effect on the IL-6-stimulated expression of $S A A 1$ or $S A A 2$ per se (Fig. 3c). However, AICAR- and metformin-induced inhibition on IL-6-stimulated expression of $S A A 1$ and $S A A 2$ was significantly lower in cells where AMPK was downregulated by siRNA (Fig. 3d,e), indicating that the anti-inflammatory effect of the agonists is dependent on AMPK. The inhibition by AICAR and metformin on IL-6induced expression of $S A A$ was not fully lost in cells transfected with siRNA against AMPK $\alpha$, which is likely to reflect the fact that AMPK activity was not fully abolished by siRNA (Fig. 3a,b). However, the activation of antiinflammatory mediators other than AMPK by AICAR and metformin cannot be excluded. The IL-6-induced expression of haptoglobin was significantly increased in cells transfected with $A M P K \alpha 1 / \alpha 2$ siRNA (Fig. 3c), suggesting the inhibitory role of AMPK activity on the expression level of this marker as well. The difference in regulation pattern of haptoglobin vs $S A A$ by AMPK is currently not understood.

To further support the role of AMPK in IL-6-stimulated gene expression, we performed siRNA transfections with LKB1, a main upstream kinase for AMPK in liver cells [28]. The reduction in AMPK activity in HepG2 cells by LKB1 siRNA transfection significantly increased the IL-6induced expression of $S A A 1, S A A 2$ and haptoglobin (see ESM Fig. 2). The fact that siRNA against AMPK $\alpha$ or LKB1 did not result in identical changes in the expression profile of acute-phase markers is probably dependent on the exact time frame and extent of inhibition of phosphoAMPK levels. Taken together, these experiments indicate the functional significance of AMPK in the repression of
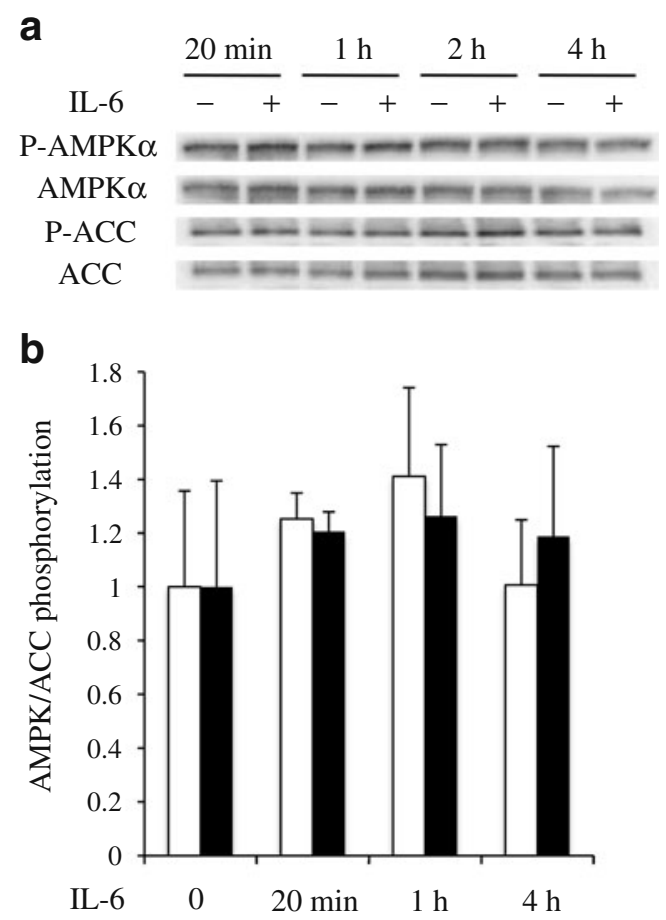

Fig. 4 AMPK activity in HepG2 cells is not regulated by IL-6. HepG 2 cells were stimulated with IL-6 for the time points indicated and cell lysates were analysed by western blot using antibodies specific for phospho-AMPK $\alpha\left(\mathrm{Thr}^{172}\right)$, total AMPK $\alpha$, phospho-ACC $\left(\mathrm{Ser}^{79}\right)$ or total ACC. a Representative western blot is shown. b Phospho-AMPK (white bars) and phospho-ACC (black bars) levels were analysed by densitometry and shown as bar histogram. Phosphorylation of AMPK and ACC in basal state is set to 1. The data are shown as means \pm SEM of three or four western blot analysis and the error bars in non-stimulated group represent the average SEM for the non-treated groups over the four time points 
IL-6-stimulated expression of acute-phase markers in liver cells.

AMPK activity in human liver cells is not directly modulated by proinflammatory cytokine action Previously, the activity of AMPK measured at the level of phosphorylation of $\operatorname{Thr}^{172}$ at the $\alpha$ subunit has been shown to be enhanced by IL-6 stimulation in skeletal muscle and adipose tissue in vitro $[19,29,30]$, whereas TNF- $\alpha$ signalling has been demonstrated to suppress AMPK activity [31]. In this study, no significant effect of the proinflammatory cytokines IL-6, TNF- $\alpha$ and IL- $1 \beta$ on the phosphorylation of the $\mathrm{Thr}^{172}$ residue of the AMPK $\alpha$ subunit or the $\mathrm{Ser}^{79}$ residue of the downstream target ACC, was observed in the liver cells (Fig. 4; ESM Fig. 1a, data not shown).

IL-6-stimulated phosphorylation of STAT3 in HepG2 cells is regulated by AMPK To characterise the molecular pathways responsible for AMPK's regulation of intracellular IL-6

a

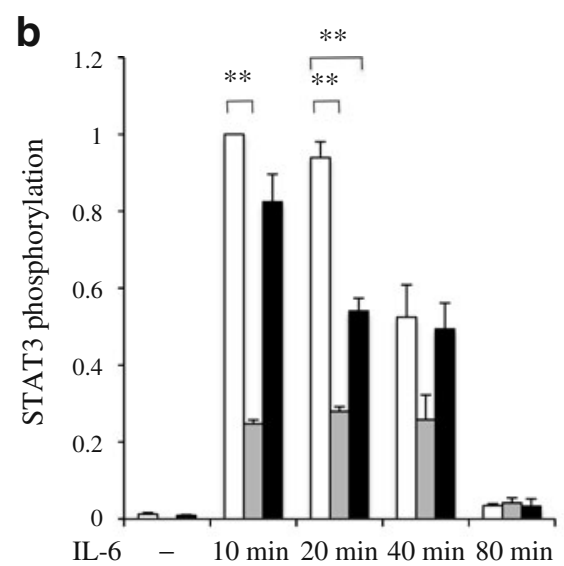

C

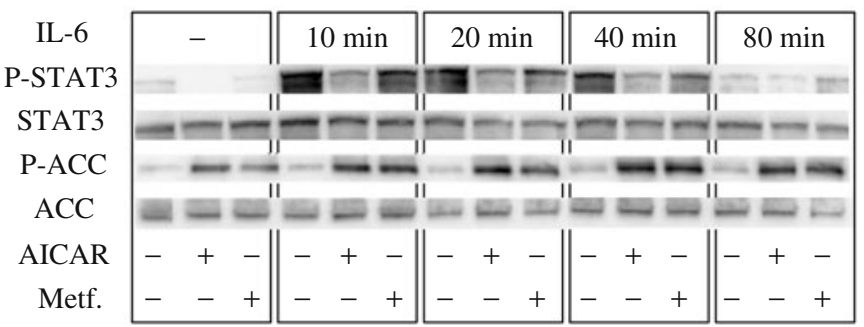

IL-6 - $10 \mathrm{~min} 20 \mathrm{~min} 40 \mathrm{~min} 80 \mathrm{~min}$
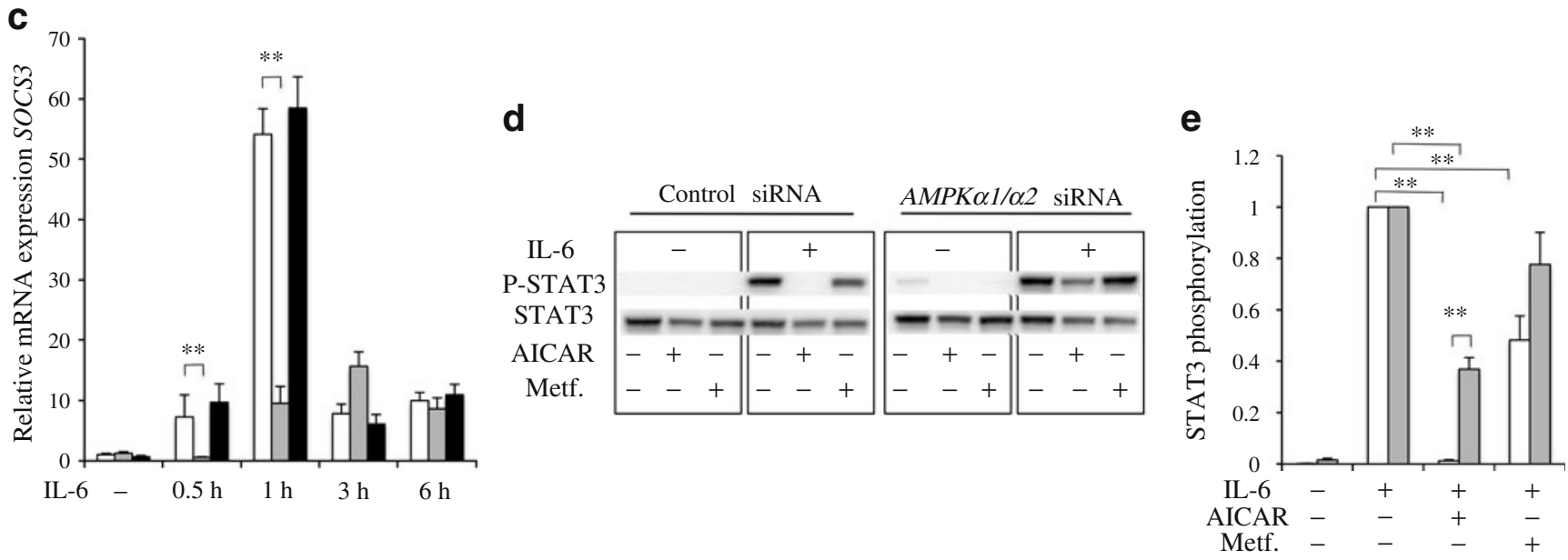

Fig. 5 IL-6-stimulated phosphorylation of STAT3 in HepG2 cells is regulated by AMPK. a HepG2 cells were cultured with AICAR ( $2 \mathrm{mmol} / \mathrm{l})$ or metformin $(5 \mathrm{mmol} / \mathrm{l})$ for $16 \mathrm{~h}$, followed by stimulation with IL- 6 for the time points indicated. Cell lysates were analysed by western blot using antibodies specific for phospho-STAT3 $\left(\mathrm{Tyr}^{705}\right)$, total STAT3, phospho-ACC $\left(\mathrm{Ser}^{79}\right)$ or total ACC. Representative western blot of three or four independent experiments is shown. Selected concentration of AICAR and metformin increased the phosphorylation of ACC to the same extent (5.0-fold and 4.8-fold respectively, $n=6$ ), indicating that AMPK was activated to a similar degree. b Phospho-STAT3 levels (a) were analysed by densitometry and shown as bar histogram (white bars, basal; grey bars, AICAR; black bars, metformin). The data are shown as means \pm SEM of three experiments. Phosphorylation of STAT3 in placebo group 10 min after stimulation with IL-6 is set to 1 . Although the tendency for reduced phosphorylation of STAT3 $\left(\mathrm{Tyr}^{705}\right)$ after incubation with AICAR and metformin was also seen in basal conditions (a), because of very low expression levels the quantification appeared less reliable. $\mathbf{c}$ Levels of the SOCS3 mRNA were determined by qRT-PCR after incubation with $\operatorname{AICAR}(2 \mathrm{mmol} / \mathrm{l})$ or metformin $(5 \mathrm{mmol} / \mathrm{l})$ for $16 \mathrm{~h}$ followed by stimulation with IL-6 for the time points indicated. Results are shown as means \pm SEM of three determinations with the basal expression of SOCS3 set to 1 (white bars, basal; grey bars, AICAR; black bars, metformin). d Phosphorylation of STAT3 $\left(\mathrm{Tyr}^{705}\right.$ ) after treatment with AICAR $(1 \mathrm{mmol} / \mathrm{l})$ or metformin $(2 \mathrm{mmol} / \mathrm{l})$ for $16 \mathrm{~h}$ in HepG2 cells transfected with $A M P K \alpha 1 / \alpha 2$ or control siRNA with and without stimulation of IL-6 (20 min) was analysed by western blot. AntiSTAT3 antibodies were used to demonstrate that no differences in total protein levels were observed. e Phospho-STAT3 levels (d) were analysed by densitometry and shown as bar histogram (white bars, Control siRNA; grey bars, $A M P K \alpha 1 / \alpha 2$ siRNA). The data are shown as means \pm SEM of three experiments. Phosphorylation of STAT3 in response to IL-6 is set to 1 . Metf, metformin. ${ }^{*} p<0.05,{ }^{*} p<0.01$ 
signalling, we quantified the phosphorylation of one of the prime downstream targets of the IL- 6 receptor complexSTAT3. As reported previously [32], IL-6 induced a rapid and transient activation of STAT3 through phosphorylation of $\operatorname{Tyr}^{705}$ (Fig. 5a,b). Treatment with AICAR and metformin markedly reduced the IL-6-stimulated induction of tyrosine phosphorylation of STAT3 in HepG2 cells (Fig. 5a,b). In addition, AICAR and metformin treatment inhibited STAT3 phosphorylation in basal as well as IL-6-stimulated conditions in primary mouse hepatocytes (see ESM Fig. 3). The effect of AICAR and metformin on phosphorylation of STAT3 was dependent on AMPK activity because the regulation was markedly reduced when AMPK expression was inhibited by anti- $\alpha 1 / \alpha 2$ siRNA in HepG2 cells (Fig. 5d,e). The effect of metformin on phosphorylation of STAT3 was less pronounced compared with the effect of AICAR (Fig. 5a,b,d,e). As the selected concentration of metformin did not seem to have a less pronounced effect on repressing the expression of $S A A 1, S A A 2$ and haptoglobin, compared with the concentration range of AICAR that was tested (Fig. 2b,c), the signalling pathways of metformin and AICAR in regulation of proinflammatory gene expression may only partially overlap and additional mechanisms other than downregulation of phospho-STAT3 may be involved in the action of metformin.

The gene encoding suppressor of cytokine signalling 3 (SOCS3) is known to be a direct target of STAT3 signalling [33]. SOCS proteins are believed to play a role in the negative feedback control of cytokine signalling [34]. In addition, SOCS3 inhibits insulin signalling as shown both in vitro and in animal models [35-37]. As reported previously, in this study IL-6 upregulated SOCS3 mRNA in HepG2 cells with the peak at $1 \mathrm{~h}$ post-induction [35] (Fig. 5c). As expected, based on the inhibition pattern of STAT3 phosphorylation, the IL-6-induced expression of SOCS3 was significantly blunted by AICAR. Within the timeframe tested, no inhibition by metformin was observed, which is consistent with the less pronounced effect of metformin on STAT3 phosphorylation (Fig. 5c).

\section{Discussion}

Development of insulin resistance in type 2 diabetes has recently been hypothesised to involve the acute-phase response [38]. This is primarily due to the strong correlation identified between increased levels of proinflammatory cytokines IL- 6 , TNF- $\alpha$ and IL-1 $\beta$ and insulin resistance $[39,40]$. Of these proinflammatory cytokines, circulating IL-6 levels have the strongest correlation with insulin resistance $[39,41]$. IL- 6 is highly produced by the adipose tissue and IL- 6 levels are increased twofold to threefold in patients with obesity, insulin resistance and type 2 diabetes [42, 43]. In addition, genetic studies in individuals with insulin resistance have identified a correlation between reduced insulin sensitivity and an allele of the $I L-6$ (also known as ILO) gene, which associates with increased expression of the gene [44]. At the same time, physical exercise promotes secretion of IL-6 from skeletal muscle [45], while improving insulin sensitivity. Further, acute IL-6 administration at physiological concentrations in healthy humans does not impair whole body glucose disposal or net leg glucose uptake [46], whereas blocking IL-6 in patients with rheumatoid arthritis leads to an enhanced plasma glucose level [47]. Taken together, the role of IL-6 in whole body energy homeostasis remains partly contradictory. However, several experimental studies have shown that IL-6 impairs insulin sensitivity and action in rodent and human hepatocytes as well as inducing hepatic insulin resistance in vivo in mice [48-50]. This insulin desensitising effect in liver cells is, in part, the result of IL-6 inducing marked inflammation in this tissue [29]. For this reason novel strategies that could counteract the IL6-stimulated inflammation in liver are of great therapeutic interest.

We examined the possible role of AMPK in the regulation of the inflammatory processes induced by cytokine action in cultured human liver cells. The results clearly support the conclusion that AMPK activation suppresses the proinflammatory gene expression induced by IL- 6 stimulation in the human hepatocarcinoma cell line HepG2. The AMPK agonists AICAR and metformin markedly blunt the IL-6-stimulated expression of $S A A$ cluster genes as well as haptoglobin in a dose-dependent manner (Fig. 2; ESM Fig. 1). Although the primary role of AICAR is activating AMPK, AICAR has been reported to affect additional AMP-regulated enzymes in liver cells, such as glucokinase and fructose 1,6-bisphosphate [51]. Also,

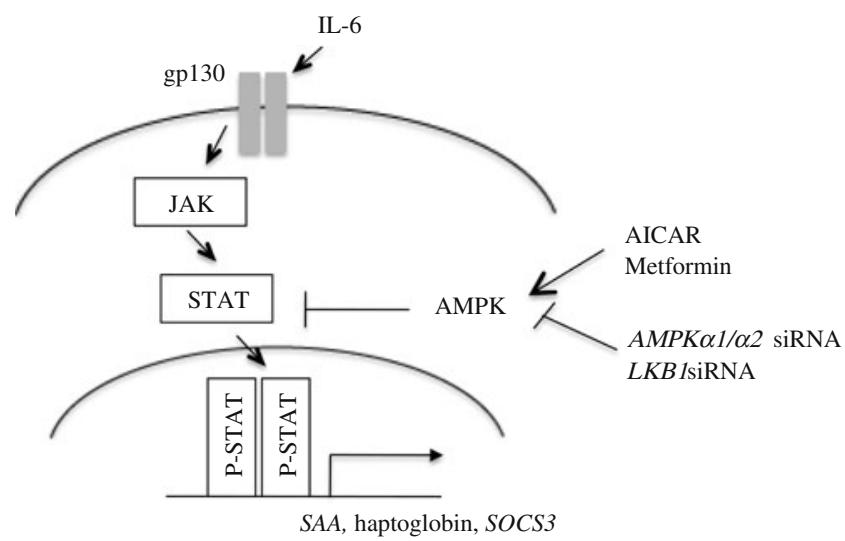

Fig. 6 Schematic model of AMPK regulation of IL-6-driven expression of proinflammatory gene expression in human liver cells. IL-6/gp130/janus kinase (JAK)/STAT signalling pathway is summarised as previously described by Heinrich et al. [33] 
metformin is a non-specific AMPK activator with the potential to activate other enzymes regulated by the cellular energy status [27]. In this study, a correlation between the time frame and concentrations of AICAR and metformin activating AMPK and the fold-reduction in expression of acute-phase markers $S A A 1, S A A 2$ and haptoglobin in response to IL-6, was observed (Fig. 2, ESM Fig. 1), indicating that the effect of the agonists on cytokine-induced gene expression is likely to be mediated by AMPK. Futhermore, the downregulation of the catalytic subunits of AMPK by siRNA significantly reversed the inhibition of $S A A 1$ and SAA2 expression by both AICAR and metformin (Fig. 3). Moreover, the reduction in AMPK activity by siRNA transfections against the main upstream kinase in liver LKB1 significantly increased the IL-6-induced expression of acute-phase markers (ESM Fig. 2). Taken together, these data indicate the functional significance of AMPK in the repression of proinflammatory gene expression in liver cells in response to IL-6 stimulation.

In addition to AMPK being a counter-regulator of IL-6stimulated inflammation, the activation of AMPK was also associated with reduction in IL- $1 \beta$-induced and TNF- $\alpha$ induced acute-phase gene expression, suggesting that AMPK may be a general promoter of an anti-inflammatory phenotype in liver cells, regardless of the initial signal. We find no evidence for IL-6, IL- $1 \beta$ or TNF- $\alpha$ directly regulating AMPK activity in HepG2 cells (Fig. 4), suggesting that AMPK is not part of the mechanisms for proinflammatory cytokines to terminate their own signal transduction.

AMPK appears to regulate IL-6 signalling in HepG2 cells as well as in primary mouse hepatocytes by directly inhibiting the activation of the main downstream target of IL-6, STAT3. IL-6 signalling is initiated by the interaction of IL-6 with a receptor complex containing the signal transducer glycoprotein 130 (gp130). This results in the activation of Janus kinases, phosphorylating gp130, which in turn results in the recruitment of STAT family members, mainly STAT3. Janus kinases phosphorylate STAT3 at $\mathrm{Tyr}^{705}$, which is crucial for nuclear translocation of STAT3 and for activation of its transcription of downstream targets such as $S A A$ and haptoglobin [33] (Fig. 6). Here, for the first time, we demonstrate that IL-6-stimulated phosphorylation of STAT3 is repressed by treatment with both AICAR and metformin, and this inhibition is dependent on the presence of AMPK activity (Fig. 5). Hence, repression of STAT3 phosphorylation may provide a mechanism for AMPK-mediated inhibition of IL-6 signalling (Fig. 6).

Previously, the increase in STAT3 in liver cells was shown to suppress gluconeogenetic gene expression and so reduce blood glucose concentrations [52]. Hepatic STAT3 overproduction also increased the circulating levels of lipids by increasing lipogenic enzymes while reducing lipid oxidation [53]. AMPK activation in liver suppresses liver gluconeogenesis and lipid production, while increasing lipid oxidation [7]. It remains to be studied if the repression of STAT3 activation may, under certain circumstances, be involved in mediating the effect of AMPK on lipid metabolism in liver.

In line with marked inhibition of STAT3 activation, AICAR also repressed IL-6-stimulated expression of SOCS3 mRNA, a direct target of STAT3. Overexpression of SOCS3 in liver leads to insulin resistance, whereas suppression of SOCS3 improves insulin sensitivity in mouse models of diabetes [37]. Correspondingly, induction of SOCS3 expression has been proposed as one mechanism of IL-6-mediated insulin resistance in liver and it is interesting to speculate that repression of SOCS3 expression might be involved in the regulation of insulin sensitivity in liver by AMPK.

Liver is a central organ in the maintenance of the glucose homeostasis of the body and, therefore, understanding the molecular mechanisms governing hepatic metabolism and inflammation are of great clinical relevance. In this study, we demonstrate that the well-characterised metabolic master switch AMPK, known to regulate both gluconeogenesis and lipid oxidation in the liver [7], also inhibits cytokine-induced inflammation in this tissue. Consequently, AMPK may represent an important point of convergence of metabolic and inflammatory signals in the liver, and may provide a novel target for developing new pharmacological strategies for a more comprehensive treatment regime of metabolic abnormalities in this tissue.

Acknowledgements This work was supported by grants from the Swedish Research Council, the Swedish Medical Society, Stiftelsen Längmanska Kulturfonden, the Swedish Foundation for Strategic Research (SSF), Johan and Jakob Söderbergs Foundation, Novo Nordisk Foundation and the European Foundation for the Study of Diabetes/Lilly research grant.

Duality of interest The authors declare that there is no duality of interest associated with this manuscript.

\section{References}

1. Hotamisligil GS (2006) Inflammation and metabolic disorders. Nature 444:860-867

2. Wellen KE, Hotamisligil GS (2005) Inflammation, stress, and diabetes. J Clin Invest 115:1111-1119

3. Carling D (2005) AMP-activated protein kinase: balancing the scales. Biochimie 87:87-91

4. Hawley SA, Boudeau J, Reid JL et al (2003) Complexes between the LKB1 tumor suppressor, STRAD alpha/beta and MO25 alpha/ beta are upstream kinases in the AMP-activated protein kinase cascade. J Biol 2:28

5. Woods A, Dickerson K, Heath R et al (2005) $\mathrm{Ca}^{2+} /$ calmodulindependent protein kinase kinase-beta acts upstream of AMPactivated protein kinase in mammalian cells. Cell Metab 2:21-33 
6. Scott JW, Hawley SA, Green KA et al (2004) CBS domains form energy-sensing modules whose binding of adenosine ligands is disrupted by disease mutations. J Clin Invest 113:274-284

7. Long YC, Zierath JR (2006) AMP-activated protein kinase signaling in metabolic regulation. J Clin Invest 116:1776-1783

8. Zhao X, Zmijewski JW, Lorne E et al (2008) Activation of AMPK attenuates neutrophil proinflammatory activity and decreases the severity of acute lung injury. Am J Physiol Lung Cell Mol Physiol 295:L497-L504

9. Kim TB, Kim SY, Moon KA et al (2007) Five-aminoimidazole-4carboxamide-1-beta-4-ribofuranoside attenuates poly(I:C)-induced airway inflammation in a murine model of asthma. Clin Exp Allergy 37:1709-1719

10. Carter AM, Bennett CE, Bostock JA, Grant PJ (2005) Metformin reduces $\mathrm{C}$-reactive protein but not complement factor $\mathrm{C} 3$ in overweight patients with Type 2 diabetes mellitus. Diabet Med 22:1282-1284

11. Mohanty P, Aljada A, Ghanim H et al (2004) Evidence for a potent antiinflammatory effect of rosiglitazone. J Clin Endocrinol Metab 89:2728-2735

12. Hattori Y, Suzuki K, Hattori S, Kasai K (2006) Metformin inhibits cytokine-induced nuclear factor kappaB activation via AMPactivated protein kinase activation in vascular endothelial cells. Hypertension 47:1183-1188

13. Peairs A, Radjavi A, Davis S et al (2009) Activation of AMPK inhibits inflammation in MRL/lpr mouse mesangial cells. Clin Exp Immunol 156:542-551

14. Kuo CL, Ho FM, Chang MY, Prakash E, Lin WW (2008) Inhibition of lipopolysaccharide-induced inducible nitric oxide synthase and cyclooxygenase- 2 gene expression by 5aminoimidazole-4-carboxamide riboside is independent of AMPactivated protein kinase. J Cell Biochem 103:931-940

15. Jhun BS, Jin Q, Oh YT et al (2004) 5-Aminoimidazole-4carboxamide riboside suppresses lipopolysaccharide-induced TNF-alpha production through inhibition of phosphatidylinositol 3-kinase/Akt activation in RAW 264.7 murine macrophages. Biochem Biophys Res Commun 318:372-380

16. Sag D, Carling D, Stout RD, Suttles J (2008) Adenosine 5'monophosphate-activated protein kinase promotes macrophage polarization to an anti-inflammatory functional phenotype. J Immunol 181:8633-8641

17. Miller EJ, Li J, Leng L et al (2008) Macrophage migration inhibitory factor stimulates AMP-activated protein kinase in the ischaemic heart. Nature 451:578-582

18. Watt MJ, Dzamko N, Thomas WG et al (2006) CNTF reverses obesity-induced insulin resistance by activating skeletal muscle AMPK. Nat Med 12:541-548

19. Carey AL, Steinberg GR, Macaulay SL et al (2006) Interleukin-6 increases insulin-stimulated glucose disposal in humans and glucose uptake and fatty acid oxidation in vitro via AMPactivated protein kinase. Diabetes 55:2688-2697

20. Kelly M, Keller C, Avilucea PR et al (2004) AMPK activity is diminished in tissues of IL-6 knockout mice: the effect of exercise. Biochem Biophys Res Commun 320:449-454

21. Mahlapuu M, Johansson C, Lindgren K et al (2004) Expression profiling of the gamma-subunit isoforms of AMP-activated protein kinase suggests a major role for gamma3 in white skeletal muscle. Am J Physiol Endocrinol Metab 286:E194-E200

22. Carlsson L, Nilsson I, Oscarsson J (1998) Hormonal regulation of liver fatty acid-binding protein in vivo and in vitro: effects of growth hormone and insulin. Endocrinology 139:2699-2709

23. Cheung PC, Salt IP, Davies SP, Hardie DG, Carling D (2000) Characterization of AMP-activated protein kinase gamma-subunit isoforms and their role in AMP binding. Biochem J 346(Pt 3):659-669

24. do Nascimento CO, Hunter L, Trayhurn P (2004) Regulation of haptoglobin gene expression in 3T3-L1 adipocytes by cytokines, catecholamines, and PPARgamma. Biochem Biophys Res Commun 313:702-708

25. Ramadori G, Van Damme J, Rieder H, Meyer zum Buschenfelde KH (1988) Interleukin 6, the third mediator of acute-phase reaction, modulates hepatic protein synthesis in human and mouse. Comparison with interleukin 1 beta and tumor necrosis factor-alpha. Eur J Immunol 18:1259-1264

26. Corton JM, Gillespie JG, Hawley SA, Hardie DG (1995) 5aminoimidazole-4-carboxamide ribonucleoside. A specific method for activating AMP-activated protein kinase in intact cells? Eur J Biochem 229:558-565

27. Brunmair B, Staniek K, Gras F et al (2004) Thiazolidinediones, like metformin, inhibit respiratory complex I: a common mechanism contributing to their antidiabetic actions? Diabetes 53: $1052-1059$

28. Carling D, Sanders MJ, Woods A (2008) The regulation of AMPactivated protein kinase by upstream kinases. Int J Obes (Lond) 32 (Suppl 4):S55-S59

29. Franckhauser S, Elias I, Rotter Sopasakis V et al (2008) Overexpression of $I l 6$ leads to hyperinsulinaemia, liver inflammation and reduced body weight in mice. Diabetologia 51:1306-1316

30. Ruderman NB, Keller C, Richard AM et al (2006) Interleukin-6 regulation of AMP-activated protein kinase. Potential role in the systemic response to exercise and prevention of the metabolic syndrome. Diabetes 55(Suppl 2):S48-S54

31. Steinberg GR, Michell BJ, van Denderen BJ et al (2006) Tumor necrosis factor alpha-induced skeletal muscle insulin resistance involves suppression of AMP-kinase signaling. Cell Metab 4: $465-474$

32. Andersson CX, Sopasakis VR, Wallerstedt E, Smith U (2007) Insulin antagonizes interleukin-6 signaling and is anti-inflammatory in 3T3L1 adipocytes. J Biol Chem 282:9430-9435

33. Heinrich PC, Behrmann I, Haan S, Hermanns HM, Muller-Newen G, Schaper F (2003) Principles of interleukin (IL)-6-type cytokine signalling and its regulation. Biochem J 374:1-20

34. Schmitz J, Weissenbach M, Haan S, Heinrich PC, Schaper F (2000) SOCS3 exerts its inhibitory function on interleukin-6 signal transduction through the SHP2 recruitment site of gp130. J Biol Chem 275:12848-12856

35. Senn JJ, Klover PJ, Nowak IA et al (2003) Suppressor of cytokine signaling-3 (SOCS-3), a potential mediator of interleukin-6dependent insulin resistance in hepatocytes. J Biol Chem 278: 13740-13746

36. Ueki K, Kondo T, Kahn CR (2004) Suppressor of cytokine signaling 1 (SOCS-1) and SOCS-3 cause insulin resistance through inhibition of tyrosine phosphorylation of insulin receptor substrate proteins by discrete mechanisms. Mol Cell Biol 24:5434-5446

37. Ueki K, Kondo T, Tseng YH, Kahn CR (2004) Central role of suppressors of cytokine signaling proteins in hepatic steatosis, insulin resistance, and the metabolic syndrome in the mouse. Proc Natl Acad Sci USA 101:10422-10427

38. Pickup JC, Mattock MB, Chusney GD, Burt D (1997) NIDDM as a disease of the innate immune system: association of acutephase reactants and interleukin-6 with metabolic syndrome X. Diabetologia 40:1286-1292

39. Kern PA, Ranganathan S, Li C, Wood L, Ranganathan G (2001) Adipose tissue tumor necrosis factor and interleukin-6 expression in human obesity and insulin resistance. Am J Physiol Endocrinol Metab 280:E745-E751

40. Uysal KT, Wiesbrock SM, Marino MW, Hotamisligil GS (1997) Protection from obesity-induced insulin resistance in mice lacking TNF-alpha function. Nature 389:610-614

41. Pradhan AD, Manson JE, Rifai N, Buring JE, Ridker PM (2001) C-reactive protein, interleukin 6 , and risk of developing type 2 diabetes mellitus. JAMA 286:327-334 
42. Yamada Y, Webber EM, Kirillova I, Peschon JJ, Fausto N (1998) Analysis of liver regeneration in mice lacking type 1 or type 2 tumor necrosis factor receptor: requirement for type 1 but not type 2 receptor. Hepatology 28:959-970

43. Campbell JS, Prichard L, Schaper F et al (2001) Expression of suppressors of cytokine signaling during liver regeneration. J Clin Invest 107:1285-1292

44. Cardellini M, Perego L, D'Adamo M et al (2005) C-174G polymorphism in the promoter of the interleukin-6 gene is associated with insulin resistance. Diab Care 28:2007-2012

45. Febbraio MA, Pedersen BK (2002) Muscle-derived interleukin-6: mechanisms for activation and possible biological roles. FASEB J 16:1335-1347

46. Steensberg A, Fischer CP, Sacchetti M et al (2003) Acute interleukin-6 administration does not impair muscle glucose uptake or whole-body glucose disposal in healthy humans. J Physiol 548:631-638

47. Choy EH, Isenberg DA, Garrood T et al (2002) Therapeutic benefit of blocking interleukin-6 activity with an anti-interleukin-6 receptor monoclonal antibody in rheumatoid arthritis: a random- ized, double-blind, placebo-controlled, dose-escalation trial. Arthritis Rheum 46:3143-3150

48. Klover PJ, Zimmers TA, Koniaris LG, Mooney RA (2003) Chronic exposure to interleukin-6 causes hepatic insulin resistance in mice. Diabetes 52:2784-2789

49. Kim HJ, Higashimori T, Park SY et al (2004) Differential effects of interleukin- 6 and -10 on skeletal muscle and liver insulin action in vivo. Diabetes 53:1060-1067

50. Senn JJ, Klover PJ, Nowak IA, Mooney RA (2002) Interleukin-6 induces cellular insulin resistance in hepatocytes. Diabetes 51:3391-3399

51. Vincent MF, Bontemps F, Van den Berghe G (1992) Inhibition of glycolysis by 5 -amino-4-imidazolecarboxamide riboside in isolated rat hepatocytes. Biochem J 281(Pt 1):267-272

52. Inoue H, Ogawa W, Asakawa A et al (2006) Role of hepatic STAT3 in brain-insulin action on hepatic glucose production. Cell Metab 3:267-275

53. Kinoshita S, Ogawa W, Okamoto Y et al (2008) Role of hepatic STAT3 in the regulation of lipid metabolism. Kobe J Med Sci 54: E200-E208 